\title{
RELAÇÃO ENTRE ABSORÇÃO ÓPTICA E FOTOCATALÍTICA DE FILMES DOS ÓXIDOS DE TITÂNIO E TUNGSTÊNIO
}

\author{
Luana Góes Soares da Silva ${ }^{1}$ \\ Annelise Kopp Alves ${ }^{2}$
}

RESUMO: A percepção da cor em vidros inorgânicos ocorre através da introdução de íons de elementos de transição ou terras raras. Ocorre enquanto o vidro ainda está no estado fundido. $\mathrm{Na}$ absorção óptica, a radiação luminosa é absorvida por transições da banda de valência para a banda de condução, onde uma parte é reemitida em comprimentos de onda específicos causados pelos níveis aumentados pelas impurezas. Nesse contexto, sintetizamos filmes contendo óxidos de titânio e tungstênio por spin-coating. Caracterizamos essas amostras por difração de raios X (XRD), microscopia eletrônica de varredura (MEV) e transmissão (TEM), fotocatálise heterogênea e testes colorimétricos. Os resultados indicam que os filmes sintetizados apresentam boas propriedades ópticas e fotocatalíticas, possivelmente devido à ocorrência de fenômenos semelhantes. A elevação da temperatura de tratamento térmico possibilitou que as vacâncias de $\mathrm{O}_{2}$ adquirissem a mobilidade necessária para passar a um estado desordenado na rede, aumentando a capacidade de degradação e absorção de luz. A presença de tungstênio aumentou a eficiência fotocatalítica dos materiais, inibiu a recombinação do par elétron/lacuna $\left[\left(e^{-}\right) /(h+)\right]$, permitindo a transferência de cargas entre o $\mathrm{TiO}_{2}$ e o $\mathrm{WO}_{3}$.

Palavras-chave: Absorção Óptica, Fotoatividade, Filmes.

ABSTRACT: The perception of color in inorganic glass occurs through the introduction of ions from transition elements or rare earths. It occurs while the glass is still in the molten state. In optical absorption, light radiation is absorbed by transitions from the valence band to the conduction band, where a part is re-emitted at specific wavelengths caused by the levels increased by impurities. In this context, we synthesized films containing titanium and tungsten oxides by spin-coating. We characterized these samples by X-ray diffraction (XRD), scanning electron microscopy (SEM) and transmission (TEM), heterogeneous photocatalysis and

\footnotetext{
Formação acadêmica mais alta: Doutora em Engenharia- Área de Concentração: Ciência e Tecnologia dos Materiais Instituição de atuação atual: Universidade Federal do Rio Grande do Sul. Email: lugoes.soares@gmail.com.

2 Formação acadêmica mais alta: Doutora em Engenharia- Área de Concentração: Ciência e Tecnologia dos Materiais Instituição de atuação atual: Universidade Federal do Rio Grande do Sul. E-mail: annelise.alves@ufrgs.br.
} 
colorimetric tests. The results indicate that the synthesized films have good optical and photocatalytic properties, possibly due to the occurrence of similar phenomena. The increase in the temperature of the heat treatment allowed the $\mathrm{O}_{2}$ vacancies to acquire the mobility necessary to pass to a disordered state in the network, increasing the degradation and light absorption capacity. The presence of tungsten increased the photocatalytic efficiency of the materials, inhibited the recombination of the pair electron/hole $\left[\left(e^{-}\right) /\left(h_{+}\right)\right]$, allowing the transfer of charges between $\mathrm{TiO}_{2}$ and $\mathrm{WO}_{3}$.

Keywords: Optical Absorption. Photoactivity. Films.

\section{INTRODUÇÃO}

As propriedades ópticas dizem respeito à resposta de um material à interação com a radiação eletromagnética, especialmente com a luz visível. O comportamento óptico de um material sólido é o resultado de suas interações com a radiação eletromagnética e comprimentos de onda dentro da região visível do espectro [I].

Quando a luz incide sobre a superfície de um objeto, um ou mais fenômenos correspondentes à cor podem ocorrer, uma parte da radiação é transmitida pelo meio, outra parte é absorvida e uma última parte é refletida na interface [I].

Materiais capazes de transmitir luz com pouca absorção e reflexão são transparentes. Materiais capazes de transmitir luz, mas de forma difusa, dispersos dentro do material, são translúcidos. Os materiais opacos são impermeáveis à transmissão da luz visível [I].

Quando a luz incide sobre a superfície de um objeto, um ou mais fenômenos correspondentes à cor podem ocorrer, uma parte da radiação é transmitida pelo meio, outra parte é absorvida e uma última parte é refletida na interface [I].

Materiais capazes de transmitir luz com pouca absorção e reflexão são transparentes. Já os materiais capazes de transmitir luz, mas de forma difusa, dispersos dentro do material, são translúcidos. E por fim, materiais opacos são impermeáveis à transmissão da luz visível [I].

O fotocromismo é um fenômeno capaz de modificar reversivelmente suas propriedades ópticas quando exposto à radiação eletromagnética, ou seja, UV visível 
ou infravermelho. Em alguns óxidos, esse fenômeno pode ser percebido quando são expostos à luz visível $[2,3,4]$.

Um material fotocrômico altera suas propriedades ópticas ao receber alguns estímulos, tais como: o campo elétrico, a radiação eletromagnética, a diferença de potencial, a temperatura, a mudança de $\mathrm{pH}$ e a pressão mecânica são algumas formas de ativar opticamente esses materiais $[2,3,4]$.

Diversos materiais têm sido utilizados no estudo do fotocromismo, principalmente óxidos de metais de transição, como: $\mathrm{MoO}_{3}, \mathrm{WO}_{3}, \mathrm{TiO}_{2}, \mathrm{~V}_{2} \mathrm{O}_{5}$ e $\mathrm{Nb}_{2} \mathrm{O}_{5}$, entre outros $[2,3,5]$.

Os metais geralmente opacos devido à luz que incide sobre eles com frequências na faixa do visível, que excita os elétrons para estados desocupados acima do nível de Fermi. Os isoladores elétricos podem ser transparentes e os semicondutores podem ser transparentes ou opacos [I].

Vários mecanismos são recomendados para descrever o efeito fotocrômico, os mais reconhecidos são: Dupla inserção de íons e elétrons, formação de centros de cor via vacâncias de $\mathrm{O}_{2}$ e smallpoloron [5].

Neste trabalho, analisamos o efeito fotocrômico por dupla inserção de íons e elétrons. Pois o fotocromismo é observado quando o material é irradiado com energia que ultrapassa a energia do band gap, onde os pares elétron-lacuna $(\mathrm{e}-/ \mathrm{h}+)$ gerados se dissociam das moléculas de $\mathrm{H}_{2} \mathrm{O}$ adsorvidas na superfície das partículas, formando $\mathrm{H}_{2}$ e $\mathrm{O}_{2}[5]$. Optamos por esse mecanismo pela ocorrência de eventos simultâneos e semelhantes na realização dos testes fotocrômicos e fotocatalíticos. O sucesso de ambos os testes está relacionado à necessidade de absorção de um fóton, para a promoção de um elétron da banda de valência para a banda de condução, superando o gap, gerando um gap na banda de valência e um elétron na banda de condução.

Assim, neste trabalho sintetizamos filmes semicondutores de $\mathrm{TiO}_{2}$ e $\mathrm{TiO}_{2}$ misturados com precursores de tungstênio $\left(\mathrm{H}_{2} \mathrm{WO}_{4}\right.$ e $\left.\mathrm{Na}_{2} \mathrm{WO}_{4} \cdot 2 \mathrm{H}_{2} \mathrm{O}\right)$ por spincoating. Nós analisamos e correlacionamos suas propriedades ópticas e fotocatalíticas na região do UV-visível. Escolhemos os precursores do $\mathrm{WO}_{3}$, por serem os que apresentam as maiores variações de absorbância óptica em todos os efeitos 
cromogênicos, o que torna esse óxido o mais utilizado em pesquisas quando se deseja estudar o efeito fotocrômico $[2,3]$.

\section{METODOLOGIA}

\section{I Obtenção das fibras através do electrospinning}

i) Preparação das soluções precursoras:

$\underline{\text { Solução de } \mathrm{TiO}_{2}}-2,5 \mathrm{ml}$ de propóxido de titânio (TiP) foram misturados; $2 \mathrm{ml}$ de ácido acético glacial e $5 \mathrm{ml}$ de uma solução alcoólica contendo ı\% em peso de polivinilpirrolidona (PVP). Esta solução foi obtida misturando io $\mathrm{g}$ do polímero (PVP) em um béquer contendo ıoo $\mathrm{mL}$ de álcool etílico, sem aquecimento e sob constante agitação magnética, até que todo o polímero estivesse completamente dissolvido.

Solução $\mathrm{TiO}_{2} / \mathrm{WO}_{3}$ - À solução contendo titânio previamente preparada, foram adicionados $\mathrm{I} \mathrm{ml}$ de peróxido de hidrogênio e o, Io $\mathrm{g}$ de $\mathrm{H}_{2} \mathrm{WO}_{4}$, os quais foram mantidos sob agitação magnética por 15 minutos.

Solução $\mathrm{TiO}_{2} / \mathrm{Na}_{2} \mathrm{WO}_{4} \cdot \underline{2} \mathrm{H}_{2} \mathrm{O}$ - $\mathrm{A}$ solução inicial contendo titânio, I ml de peróxido de hidrogênio e o, Io $\mathrm{g}$ de $\mathrm{Na}_{2} \mathrm{WO}_{4} \cdot 2 \mathrm{H}_{2} \mathrm{O}$ foram adicionados sob agitação magnética por 15 minutos.

ii) Electrospinning

Para a obtenção das fibras pelo processo de electrospinning, uma seringa de plástico de $5 \mathrm{~mL}$ conectada a uma agulha hipodérmica de aço inoxidável com diâmetro interno de I $\mathrm{mm}$ foi preenchida com a solução precursora. A agulha foi conectada à fonte de alta tensão. A distância entre a ponta da agulha e o coletor cilíndrico giratório coberto por uma folha de alumínio era de $12 \mathrm{~cm}$. Uma tensão de $13,5 \mathrm{kV}$ foi aplicada entre a agulha e o coletor. Uma bomba de infusão (KD Scientific) controlava o fluxo da solução precursora $(\mathrm{I}, 8 \mathrm{~mL} / \mathrm{h})$. As fibras foram coletadas a cada 30 minutos, durante o período de 4 horas para cada formulação.

iii) Tratamento térmico

Em seguida, as fibras foram tratadas termicamente em um forno elétrico da marca Sanchis nas temperaturas de $650^{\circ} \mathrm{C}$, $700{ }^{\circ} \mathrm{C}$, $750{ }^{\circ} \mathrm{C}$ e $800{ }^{\circ} \mathrm{C}$, com patamar de I hora e taxa de aquecimento de $\mathrm{I}, 4^{\circ} \mathrm{C} / \mathrm{min}$. A Figura I é uma 
fotografia das fibras de $\mathrm{TiO}_{2}$ obtidas por electrospinning, após o tratamento térmico.

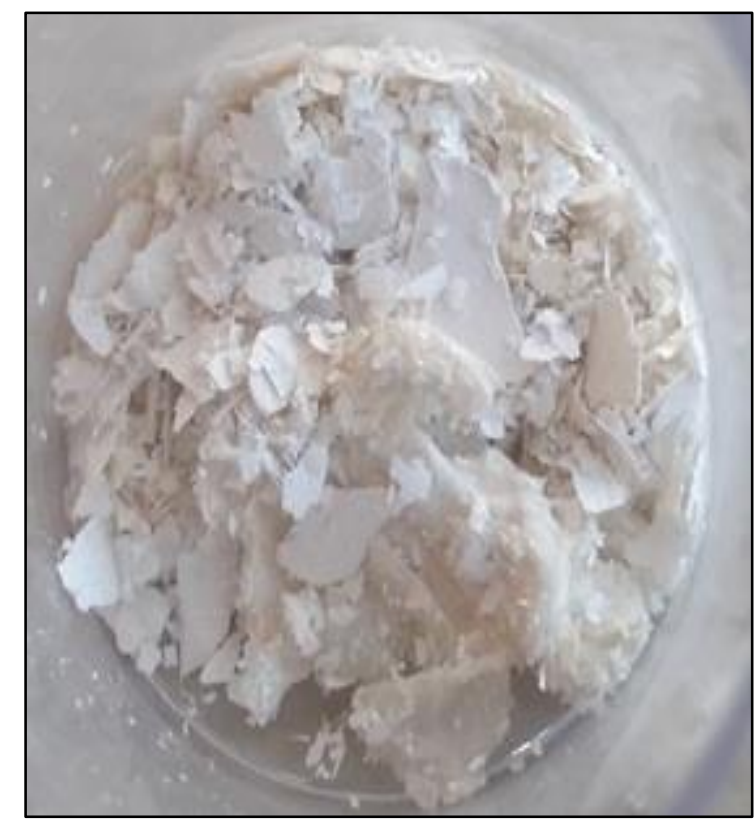

Figura I. Imagem fotográfica das fibras de $\mathrm{TiO}_{2}$ obtidas por electrospinning, após o tratamento térmico a $650^{\circ} \mathrm{C}$.

\subsubsection{Preparação das dispersões de fibras e o padrão $\mathrm{P}_{25}$}

$0,25 \mathrm{~g}$ de fibras de $\mathrm{TiO}_{2}, \mathrm{TiO}_{2} / \mathrm{WO}_{3}$ e $\mathrm{TiO}_{2} / \mathrm{Na}_{2} \mathrm{WO}_{4} \cdot 2 \mathrm{H}_{2} \mathrm{O}$, tratadas termicamente a $650^{\circ} \mathrm{C}, 700{ }^{\circ} \mathrm{C}, 750^{\circ} \mathrm{C}$ e $800{ }^{\circ} \mathrm{C}$ e o,25 g do padrão $\mathrm{TiO}_{2}-\mathrm{P}_{25}$ (pó comercial Evonik) foram misturados em $8 \mathrm{~mL}$ de anidro etanol e o,8 $\mathrm{mL}$ de acetilacetona (SigmaAldrich). As dispersões foram preparadas separadamente, totalizando 13 amostras. As misturas foram dispersas em ultrassom por io minutos. Após esse período, foram adicionados o,I $\mathrm{ml}$ de Triton X-ıoo (Sigma-Aldrich) e o,4 g de polivinilbitiral (PVB) e mantidos sob agitação magnética por ıo minutos.

\subsubsection{Spin-coating}

Os filmes foram obtidos por spin-coating, depositando 5 gotas de cada uma das dispersões previamente preparadas nas placas de vidro, dimensões $\mathrm{I} \mathrm{cm} \times 2 \mathrm{~cm}$, já recobertas com FTO (Fluorine-Doped Tin Oxide, XOP Glass). O equipamento utilizado foi um (TC ıoo Spin Coater) com rotação de 8oo RPM por $30 \mathrm{~s}$. A Figura 2 mostra os filmes obtidos pela técnica de spin-coating. 


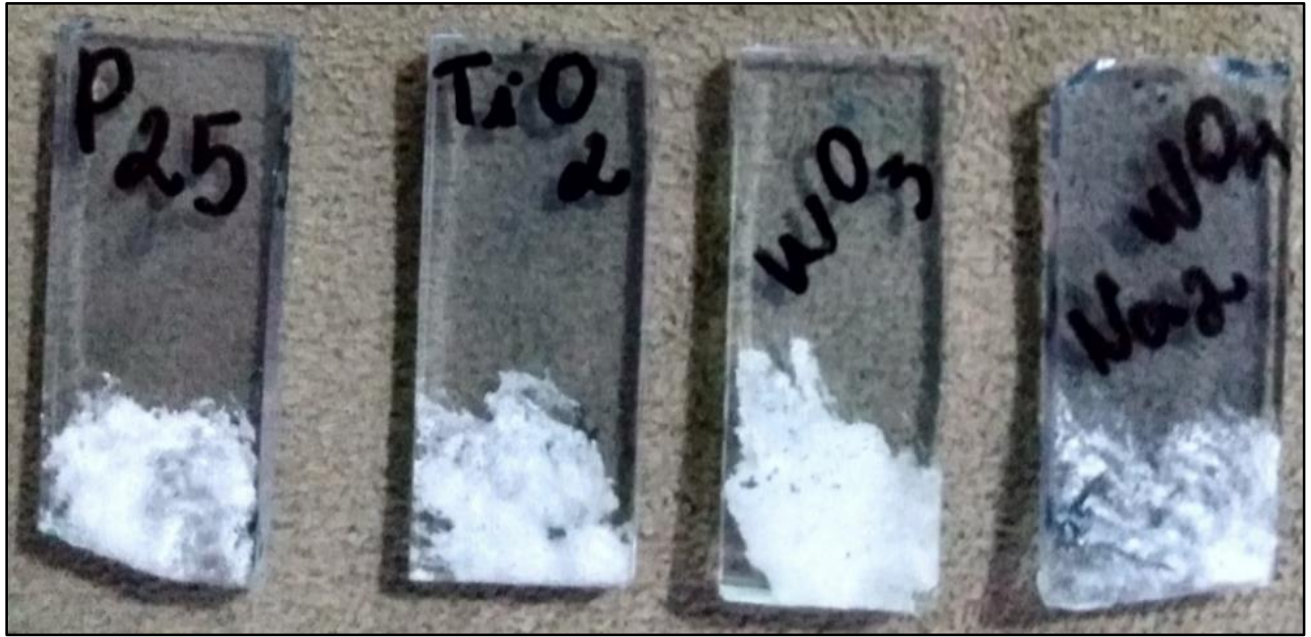

(a)

(b)

(c)

(d)

Figura 2. Filmes de (a) padrão $\mathrm{P} 25$, (b) $\mathrm{TiO}_{2}$, (c) $\mathrm{TiO}_{2} / \mathrm{WO}_{3}$ e (d) $\mathrm{TiO}_{2} / \mathrm{Na}_{2} \mathrm{WO}_{4} \cdot 2 \mathrm{H}_{2} \mathrm{O}$ obtidos por spin-coating.

\subsection{Caracterização dos materiais}

Para identificar as fases presentes nas amostras foi utilizado um difratômetro PHILIPS com radiação $\mathrm{CuK} \alpha$, na tensão de $40 \mathrm{kV}$ e $40 \mathrm{~mA}$, equipado com o software X'PERT HightScore. Para avaliar a morfologia das amostras e identificar a presença de átomos de $\mathrm{Na}, \mathrm{W}$, Ti e $\mathrm{O}$ nos filmes, dependendo da composição dos precursores, foi utilizado um microscópio eletrônico de varredura (MEV, JEOL JSM 6o6o) equipado com um detector de raios $\mathrm{X}$ característico (EDS). Para maiores detalhes sobre a microestrutura das amostras, um microscópio eletrônico de transmissão (MET, JEM i2ooExll) também foi utilizado. Um espectrofotômetro de duplo feixe UV-vis-NIR (Carysooo) foi usado para determinar o gap das amostras, através da correlação de Kubelka-Munk. A colorimetria foi analisada por um espectrofotômetro Konica-Minolta, CM 26ood, com esfera integrada e filtro ultravioleta. O iluminante usado foi o D65, e as medidas de cor simulavam um observador a ro ${ }^{\circ}$. A fotoatividade dos filmes foi analisada por um espectrofotômetro Cary Agilent 7ooo com o acessório UMA.

\section{RESULTADOS E DISCUSSÃO}

Todos os filmes antes do tratamento térmico se apresentaram amorfos. Em filmes contendo $\mathrm{TiO}_{2}$, a maior presença de nível de anatase foi observada até $700{ }^{\circ} \mathrm{C}$, 
identificada por XRD (Figura 3a). A $750{ }^{\circ} \mathrm{C}$, a presença de fase rutilo, além de anatase, provavelmente foi detectada como resultado de uma transformação de fase devido à temperatura de sinterização [6]. Já os filmes de $\mathrm{TiO}_{2} / \mathrm{WO}_{3}$ apresentaram picos a $2 \theta=23,34,23,64,24,80,32,85,34,84^{\circ}$, entre outros, relacionados à fase monoclínica do óxido de tungstênio. Foi possível reconhecer a presença das fases anatase, brookita e rutilo. Filmes de $\mathrm{TiO}_{2} / \mathrm{Na}_{2} \mathrm{WO}_{4} \cdot 2 \mathrm{H}_{2} \mathrm{O}$ exibiram as fases anatase, brookita e rutilo para $\mathrm{TiO}_{2}$, a fase monoclínica para óxido de tungstênio e a presença do grupo $\mathrm{Na}(\mathrm{OH})$, devido a presença do precursor $\mathrm{Na}_{2} \mathrm{WO}_{4} \cdot 2 \mathrm{H}_{2} \mathrm{O}$.

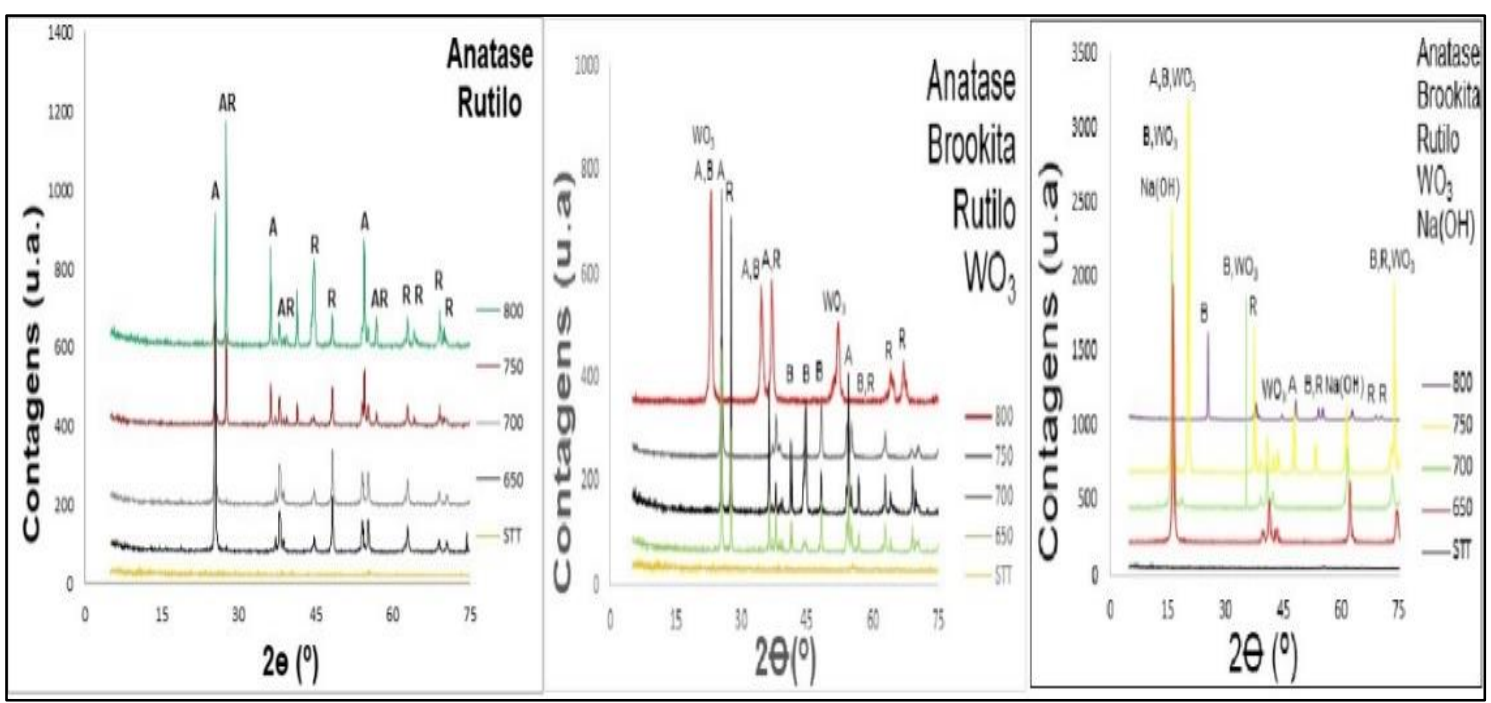

(a) (b) (c)

Figura 3. Difratograma das amostras (a) $\mathrm{TiO}_{2}$, (b) $\mathrm{TiO}_{2} / \mathrm{WO}_{3}$ e (c) $\mathrm{TiO}_{2} / \mathrm{Na}_{2} \mathrm{WO}_{4} \cdot 2 \mathrm{H}_{2} \mathrm{O}$.

As microestruturas dos filmes são mostradas na Figura 4. Em geral, as fibras apresentam uma aparência semelhante, parecem não ter uma orientação preferencial, com microestrutura alongada e superfície irregular. As imagens de MET dos filmes apresentam a presença de um aglomerado de fibras, principalmente nas formulações $\mathrm{TiO}_{2} / \mathrm{WO}_{3}$ e $\mathrm{TiO}_{2} / \mathrm{Na}_{2} \mathrm{WO}_{4} \cdot 2 \mathrm{H}_{2} \mathrm{O}$, o que pode ser atribuído à inserção do tungstênio às amostras de $\mathrm{TiO}_{2}$. Essas amostras têm fibras alongadas com superfícies irregulares. Imagens de microscopia eletrônica de transmissão (MET) mostraram que as fibras parecem ser feitas de um agrupamento axial de muitos cristais. De acordo com Lu et. al., [7] a inserção de $\mathrm{WO}_{3}$ nas fibras dá essas características altamente cristalinas. 


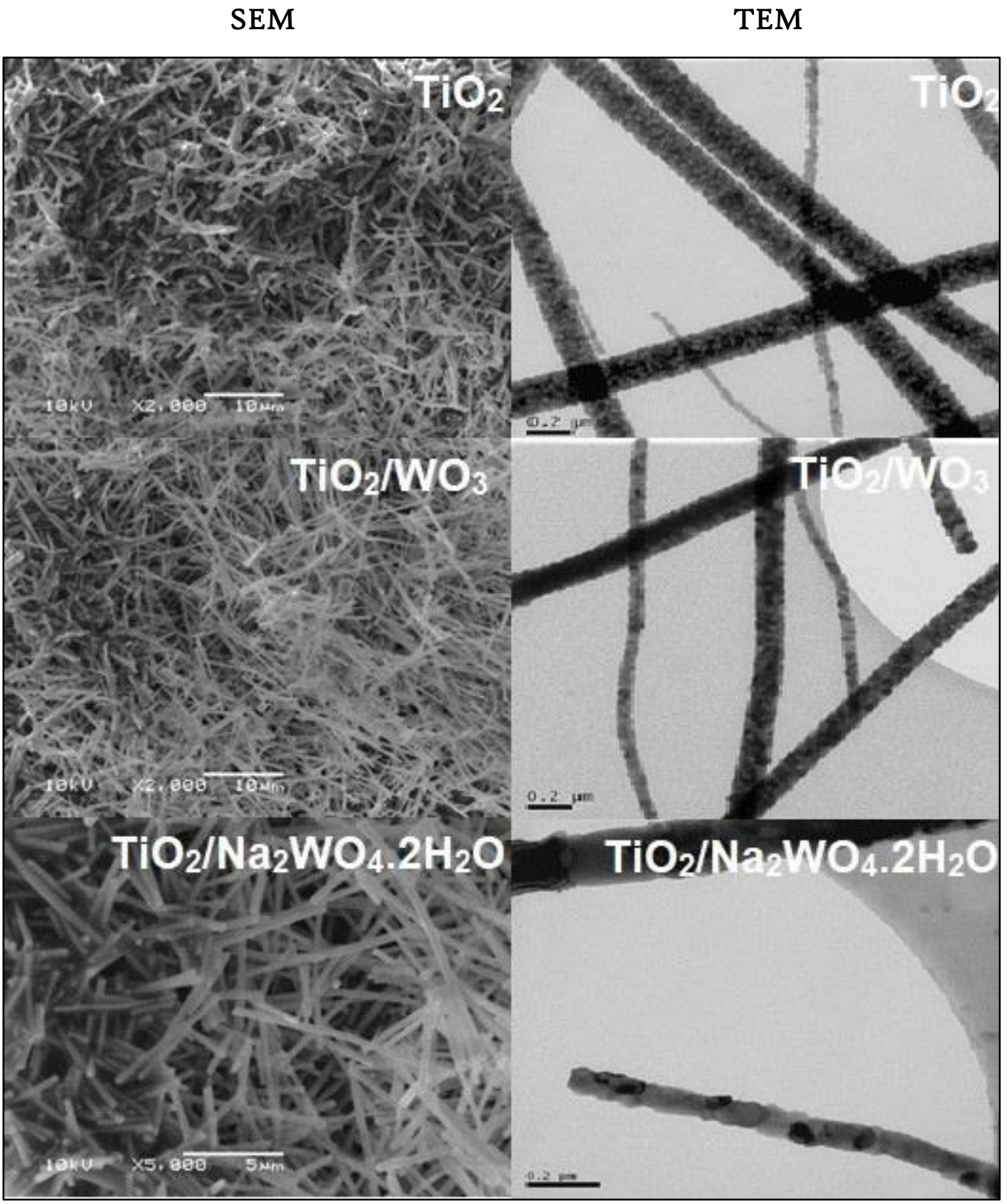

Figura 4. Microscopia eletrônica de varredura e de transmissão dos filmes de $\mathrm{TiO}_{2}, \mathrm{TiO}_{2} / \mathrm{WO}_{3}$ e $\mathrm{TiO}_{2} / \mathrm{Na}_{2} \mathrm{WO}_{4} \cdot 2 \mathrm{H}_{2} \mathrm{O}$.

As Figuras 5, 6 e 7 mostram a atividade catalítica dos filmes $\mathrm{TiO}_{2}, \mathrm{TiO}_{2} / \mathrm{WO}_{3}$ e $\mathrm{TiO}_{2} / \mathrm{Na}_{2} \mathrm{WO}_{4} \cdot 2 \mathrm{H}_{2} \mathrm{O}$ na degradação do corante laranja de metila durante 135 minutos de exposição à luz UV-A $(\mathrm{CQ}=365 \mathrm{~nm})$. 
O gráfico I mostra a fotoatividade dos filmes de $\mathrm{TiO}_{2}$ na degradação do corante alaranjado de metila. O gráfico mostra que todas as amostras foram capazes de degradar o corante. Todos os filmes mostraram atividade fotocatalítica. Os filmes de $\mathrm{TiO}_{2}$ mais fotoativos foram aqueles que receberam tratamento térmico a $650{ }^{\circ} \mathrm{C}$, conseguiram degradar aproximadamente 50\% do corante alaranjado de metila em 135 minutos de irradiação UVA. Os filmes tratados a $700{ }^{\circ} \mathrm{C}$ e o padrão $\mathrm{P}_{25}$ tiveram comportamento semelhante, degradando aproximadamente $47 \%$ do corante em 135 minutos de irradiação UVA. Os filmes tratados a $750^{\circ} \mathrm{C}$ e $800{ }^{\circ} \mathrm{C}$ degradaram aproximadamente $45 \%$ e 40\%, respectivamente, do corante em 135 minutos de irradiação UVA.

Esta diminuição observada na fotoatividade das amostras é resultado da formação da fase rutilo, que no caso dos filmes decorre de tratamentos acima de $700{ }^{\circ} \mathrm{C}$. A forma rutilo é menos fotoativa que a forma anatase e, por isso, seu aparecimento reduz a atividade fotocatalítica dos filmes sintetizados [8].

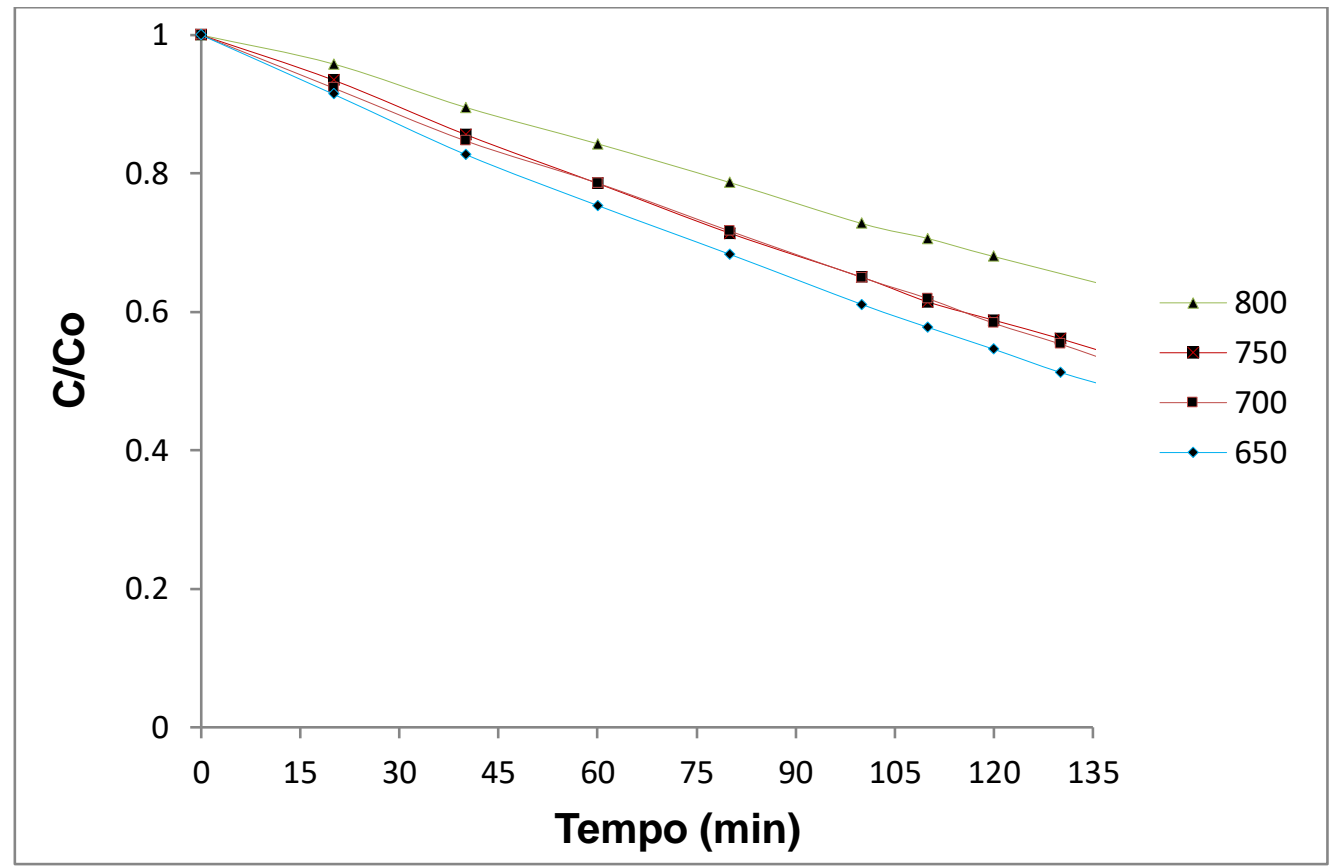

Figure 5. Fotoatividade dos filmes de $\mathrm{TiO}_{2}$ na descoloração do corante alaranjado de metila.

A presença de tungstênio $\left(\mathrm{H}_{2} \mathrm{WO}_{4}\right)$ nos filmes de $\mathrm{TiO}_{2} / \mathrm{WO}_{3}$ aumentou a atividade fotocatalítica das amostras tratadas a $700{ }^{\circ} \mathrm{C}, 750{ }^{\circ} \mathrm{C}$ e $800{ }^{\circ} \mathrm{C}$, para aproximadamente 40\%, 50\% e 90\% de degradação, respectivamente. Essa eficácia é 
devida; a elevação da temperatura do tratamento térmico e consequente redução do band gap de 2,58 eV para 2,54 eV, inibição da recombinação do par de elétrons/lacuna $\left[\left(e^{-}\right) /(h+)\right]$, o que permitiu a transferência de cargas entre os $\mathrm{TiO}_{2}$ e $\mathrm{WO}_{3}$, e o aumento na formação de defeitos específicos (vacâncias de $\mathrm{O}_{2}$ ). $\mathrm{O}$ aumento da temperatura de tratamento térmico permitiu que as vacâncias de $\mathrm{O}_{2}$ adquirissem a mobilidade necessária para passar a um estado desordenado na rede, aumentando a capacidade de degradação e a eficiência do processo [9,IO,II $]$.

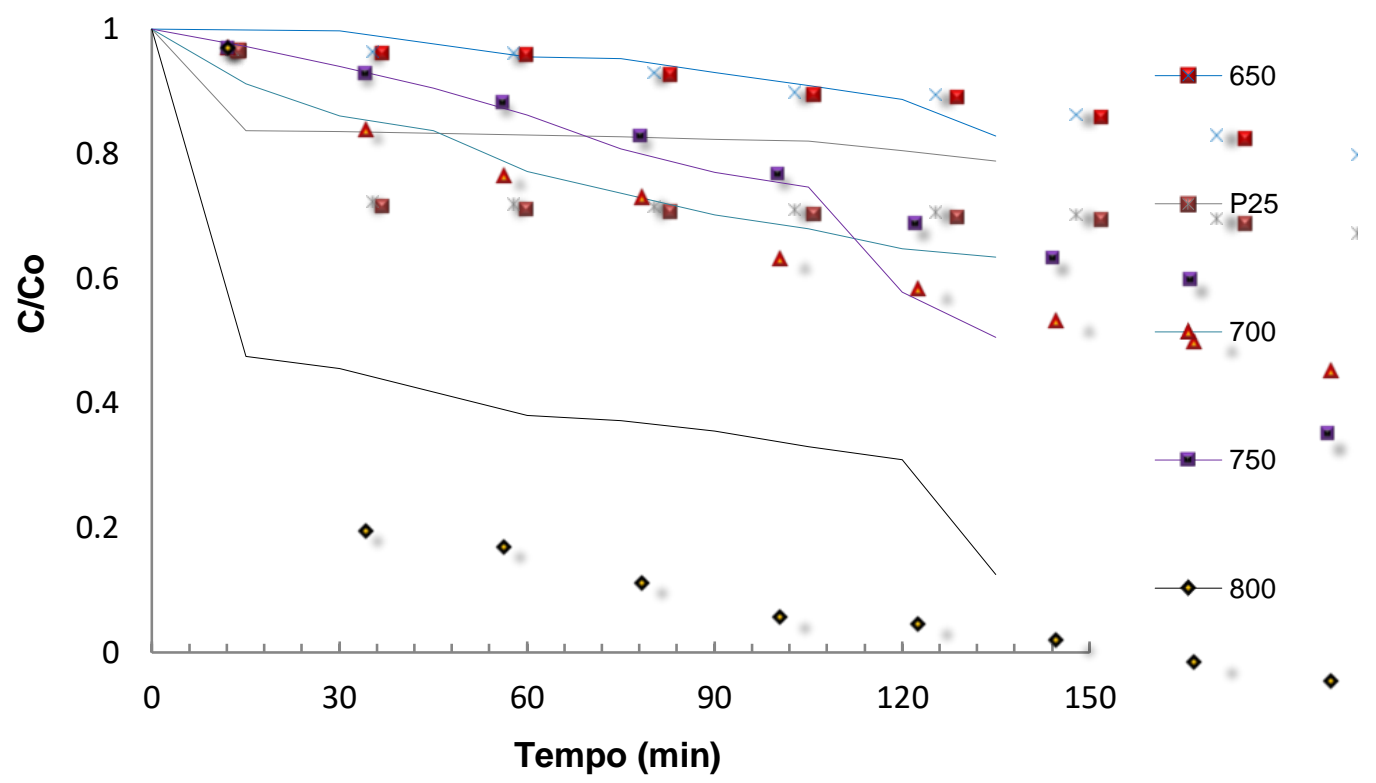

Figure 6. Fotoatividade dos filmes de $\mathrm{TiO}_{2} / \mathrm{WO}_{3}$ na descoloração do corante alaranjado de metila.

As amostras mais eficazes na degradação do corante alaranjado de metila foram os filmes contendo $\mathrm{TiO}_{2} / \mathrm{Na}_{2} \mathrm{WO}_{4} \cdot 2 \mathrm{H}_{2} \mathrm{O}$ tratados a $700 \stackrel{\circ}{\mathrm{C}}$, $750 \stackrel{\circ}{\circ} \mathrm{C}$ e $800 \stackrel{\circ}{\mathrm{C}}$, respectivamente. A Figura $7 \mathrm{~b}$ mostra a eficácia dessas amostras na descoloração do corante ao longo do tempo, são cubetas contendo alíquotas de $4 \mathrm{~mL}$ de solução aquosa contendo o corante mais a presença do catalisador (filmes), logo após o término dos testes fotocatalíticos. A Figura 7a é o resultado gráfico após essas cubetas serem analisadas por espectrofotometria. Ambas as Figuras apresentam de forma diferente a mesma informação, que é a alta capacidade dessas amostras na degradação do corante alaranjado de metila. Das 5 amostras sintetizadas, 3 degradaram aproximadamente $100 \%$ do corante em 90 minutos de exposição à radiação UV-A. As razões para a ocorrência de tal eficácia são devidas, além do 
sinergismo existente entre as fases anatase e rutilo, a redução do band gap das amostras, também a presença de sódio associada ao aumento da temperatura do tratamento térmico, que aumentou o número de defeitos (vacâncias de $\mathrm{O}_{2}$ ) na rede cristalina de $\mathrm{TiO}_{2}$. As vacâncias de $\mathrm{O}_{2}$ são defeitos pontuais que ocupam posições na rede atômica e estão fortemente vinculadas à aplicação em fotocatálise. Com a presença do sódio, o $\mathrm{TiO}_{2}$ adquiriu estabilidade da fase estrutural, responsável por aumentar a condutividade ao íon oxigênio. A elevação da temperatura do tratamento térmico possibilitou que as vacâncias de $\mathrm{O}_{2}$ adquirissem a mobilidade necessária para se mover em um estado desordenado na rede cristalina [9]. Em um estudo de Chen et. al., [12] descrevem a obtenção de nanopartículas pretas de $\mathrm{TiO}_{2}$ com excelente desempenho fotocatalítico, devido à geração de vacâncias de $\mathrm{O}_{2}$.

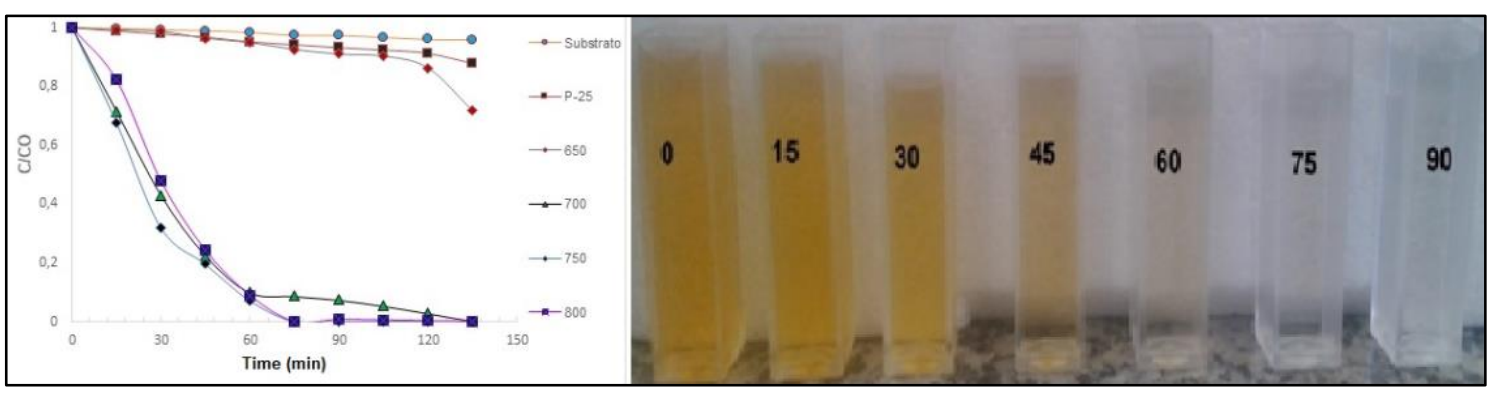

Figura 7. Fotoatividade dos filmes de $\mathrm{TiO}_{2} / \mathrm{Na}_{2} \mathrm{WO}_{4} \cdot 2 \mathrm{H}_{2} \mathrm{O}$ na descoloração do corante alaranjado de metila. (a) gráfico, resultado do teste fotocatalítico após a análise por espectrofotometria, e (b) cubetas de polimetilmetacrilato contendo a solução do corante alaranjado de metila e os semicondutores (filmes) antes da análise por espectrofotometria.

Os resultados dos testes colorimétricos realizados nos filmes de $\mathrm{P}_{25}$ (padrão), de $\mathrm{TiO}_{2}$, de $\mathrm{TiO}_{2} / \mathrm{WO}_{3}$ e de $\mathrm{TiO}_{2} / \mathrm{Na}_{2} \mathrm{WO}_{4} \cdot 2 \mathrm{H}_{2} \mathrm{O}$, são apresentados na Tabela I. Os registros para cada amostra foram obtidos com base no sistema CIE- $\mathrm{L}^{*} \mathrm{a} *$ b e a faixa de medição cobriu todo o espectro visível (400 a $700 \mathrm{~nm}$ ). A Tabela I também mostra os valores de luminescência (\% L), ou seja, a quantidade de luz que é percebida em uma determinada cor. Se a luminescência (\% L) for próxima de o\% representa a ausência total de luz refletida (preta) e se for próxima de ıoo\% representa a reflexão total da luz (branca) [13]. E os valores de $\Delta \mathrm{L}^{*}$, que informam sobre as diferenças entre as tonalidades em mais claro ou mais escuro. Os valores positivos $(+)$ de $\Delta \mathrm{L}^{*}$ indicam a cor mais clara e os valores negativos $(-)$ de $\Delta \mathrm{L}^{*}$ indicam a cor mais escura. 
Durante os testes colorimétricos, o padrão $\mathrm{P}_{25}$ e os filmes de $\mathrm{TiO}_{2}$ tiveram absorbância máxima na região da cor azul escura, influenciada pelos valores positivos de $\mathrm{a}^{*}$ (cor vermelha) e valores negativos de $\mathrm{b}^{*}$ (cor azul). A tonalidade escura das amostras foi determinada com base nos valores negativos de $\Delta L^{*}$. Os filmes de $\mathrm{TiO}_{2} / \mathrm{WO}_{3}$ tiveram absorbância máxima na região da cor azul claro, influenciada pelos valores negativos de $\mathrm{a}$ * (cor verde) e valores negativos de $\mathrm{b}$ * (cor azul). O matiz claro das amostras foi determinado com base nos valores positivos de $\Delta L^{*}$. A absorbância máxima na região da cor azul alcançada pelos filmes de $\mathrm{TiO}_{2} \mathrm{e}$ $\mathrm{TiO}_{2} / \mathrm{WO}_{3}$ já era esperada, uma vez que ambas as soluções precursoras (Figura 8a-b) são de cor amarela, diferindo apenas na tonalidade. E, nas análises colorimétricas, a absorbância máxima ocorre na região de coloração complementar, e neste caso a cor complementar ao amarelo é o azul. Por fim, os filmes de $\mathrm{TiO}_{2} / \mathrm{Na}_{2} \mathrm{WO}_{4} \cdot 2 \mathrm{H}_{2} \mathrm{O}$ apresentaram máxima transmitância de luz na região amarelo-alaranjado, influenciada pelos valores positivos de $a^{*}$ (cor vermelha) e $b^{*}$ (cor amarela). Esse resultado já era esperado, pois a solução $\mathrm{TiO}_{2} / \mathrm{Na}_{2} \mathrm{WO}_{4} \cdot 2 \mathrm{H}_{2} \mathrm{O}$ (Figura 8c) é transparente. No caso de um material transparente, todas as cores não são visíveis no filtro ou que não passam por ele são absorvidas. Com base nisso, a cor absorvida pelos filmes $\mathrm{TiO}_{2} / \mathrm{Na}_{2} \mathrm{WO}_{4} \cdot 2 \mathrm{H}_{2} \mathrm{O}$ foi a cor azul- arroxeado, que é a cor complementar ao amarelo-alaranjado [13].

Tanto o padrão $\mathrm{P}_{25}$ quanto os filmes de $\mathrm{TiO}_{2}, \quad \mathrm{TiO}_{2} / \mathrm{WO}_{3}$ e $\mathrm{TiO}_{2} / \mathrm{Na}_{2} \mathrm{WO}_{4} \cdot 2 \mathrm{H}_{2} \mathrm{O}$ apresentaram uma boa quantidade de luz percebida, de acordo com os valores de luminescência (\% L) mostrados na Tabela I.

Nos filmes sintetizados, a radiação luminosa foi absorvida por transições que ocorrem da banda de valência para a banda de condução, sendo uma parte reemitida em comprimentos de onda específicos. Isso só foi possível devido ao fato dos filmes sintetizados serem semicondutores com espaçamento de bandas na faixa de 2,24 eV e 3,24 eV (Tabela 2). Nesse caso, então, ocorreu a absorção e a transmissão, e a cor resultante resultou da interação entre as frequências transmitidas e aquelas reemitidas após a absorção. No caso dos filmes $\mathrm{TiO}_{2} / \mathrm{Na}_{2} \mathrm{WO}_{4} \cdot 2 \mathrm{H}_{2} \mathrm{O}$, oriundos de uma solução transparente, a manifestação da cor ocorreu por meio de uma absorção 
seletiva de bandas, em comprimento de onda específicos. A cor observada é o resultado da combinação dos comprimentos de onda transmitidos.

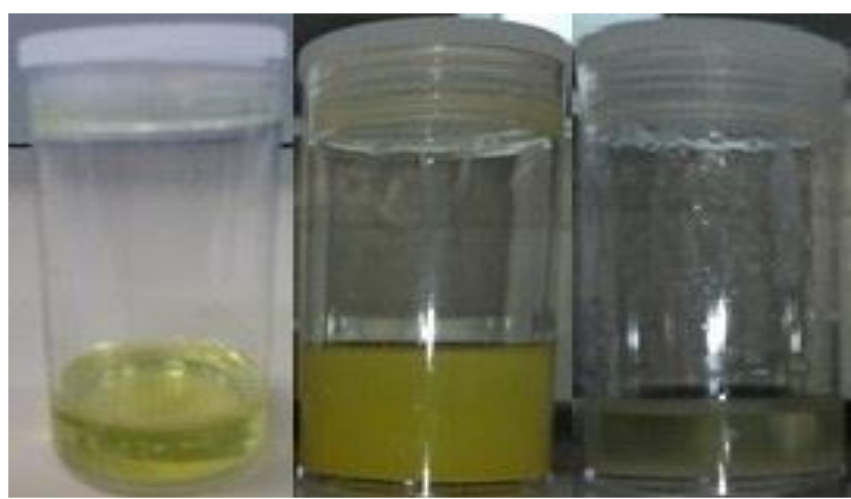

(a) (c)

Figura 8. Cor transmitida pelas soluções de (a) $\mathrm{TiO}_{2}$, (b) $\mathrm{TiO}_{2} / \mathrm{WO}_{3}$ and (c) $\mathrm{TiO}_{2} / \mathrm{Na}_{2} \mathrm{WO}_{4} \cdot 2 \mathrm{H}_{2} \mathrm{O}$.

Tabela r. Coordenadas colorimétricas dos filmes analisados pelo do sistema CIE-L*a*b.

\begin{tabular}{|c|c|c|c|c|c|}
\hline \multirow[b]{2}{*}{ Amostras } & \multicolumn{5}{|c|}{ Coordenadas Colorimétricas } \\
\hline & $a^{*}$ & $b^{*}$ & $\mathrm{~L}^{*}$ & $\begin{array}{c}\text { Diferença de } \\
\text { tonalidade } \\
\text { (Claro/escuro) } \\
\Delta \mathrm{L}^{*}\end{array}$ & Cor absorvida \\
\hline Filmes $\mathrm{TiO}_{2} 650^{\circ} \mathrm{C}$ & $+0,30$ & $-4,50$ & 83,16 & $-33,97$ & Azul-escuro \\
\hline Filmes $\mathrm{TiO}_{2} 700 \stackrel{\circ}{\circ}$ & $+0,14$ & $-5,25$ & 87,73 & $-55,23$ & Azul-escuro \\
\hline Filmes $\mathrm{TiO}_{2} 750^{\circ} \mathrm{C}$ & $+3,15$ & $-I, 57$ & 91,29 & $-22,14$ & Azul-escuro \\
\hline Filmes $\mathrm{TiO}_{2} 800 \stackrel{\circ}{\mathrm{C}}$ & $+3,43$ & $-4,62$ & 96,38 & $-40,66$ & Azul-escuro \\
\hline Filmes $\mathrm{TiO}_{2} / \mathrm{WO}_{3} 650^{\circ} \mathrm{C}$ & $-I, 46$ & $-\mathrm{I}, 99$ & 57,33 & $+64,73$ & Azul-claro \\
\hline Filmes $\mathrm{TiO}_{2} / \mathrm{WO}_{3} 700{ }^{\circ} \mathrm{C}$ & $-3,78$ & $-I, 59$ & 86,84 & $+56,73$ & Azul-claro \\
\hline Filmes $\mathrm{TiO}_{2} / \mathrm{WO}_{3} 750^{\circ} \mathrm{C}$ & $-2,97$ & $-I, 72$ & 88,46 & $+77,21$ & Azul-claro \\
\hline Filmes $\mathrm{TiO}_{2} / \mathrm{WO}_{3} 800 \stackrel{\circ}{\mathrm{C}}$ & $-1,85$ & $-2,94$ & 93,71 & $+58,48$ & Azul-claro \\
\hline $\begin{array}{l}\text { Filmes } \mathrm{TiO}_{2} / \mathrm{Na}_{2} \mathrm{WO}_{4} \cdot 2 \mathrm{H}_{2} \mathrm{O} 650 \\
\text { oC }\end{array}$ & $+\mathrm{I}, 4 \mathrm{I}$ & $+4,58$ & 86,64 & $-37,75$ & $\begin{array}{l}\text { Amarelo- } \\
\text { alaranjado }\end{array}$ \\
\hline $\begin{array}{l}\text { Filmes } \mathrm{TiO}_{2} / \mathrm{Na}_{2} \mathrm{WO}_{4} \cdot 2 \mathrm{H}_{2} \mathrm{O} 700 \\
\stackrel{\mathrm{o}}{ } \mathrm{C}\end{array}$ & $+1,37$ & +3 , I0 & 89,02 & $-55,27$ & $\begin{array}{l}\text { Amarelo- } \\
\text { alaranjado }\end{array}$ \\
\hline
\end{tabular}


Revista Ibero- Americana de Humanidades, Ciências e Educação- REASE

\begin{tabular}{|l|c|c|c|c|c|}
\hline $\begin{array}{l}\text { Filmes } \mathrm{TiO}_{2} / \mathrm{Na}_{2} \mathrm{WO}_{4} \cdot 2 \mathrm{H}_{2} \mathrm{O} 750 \\
\text { oC }\end{array}$ & $+2,55$ & $+1,22$ & 91,89 & $-26,44$ & $\begin{array}{c}\text { Amarelo- } \\
\text { alaranjado }\end{array}$ \\
\hline $\begin{array}{l}\text { Filmes } \mathrm{TiO}_{2} / \mathrm{Na}_{2} \mathrm{WO}_{4} \cdot 2 \mathrm{H}_{2} \mathrm{O} 800 \\
\text { o }\end{array}$ & $+2,16$ & $+5,5 \mathrm{I}$ & 94,36 & $-42,68$ & $\begin{array}{c}\text { Amarelo- } \\
\text { alaranjado }\end{array}$ \\
\hline Filmes $\mathrm{TiO}_{2}-\mathrm{P} 25$ padrão & $+0,36$ & $-4,43$ & 93,27 & $-42,53$ & Azul-escuro \\
\hline
\end{tabular}

Tabela 2. Valores de band gap dos filmes sintetizados por spin-coating.

\begin{tabular}{|c|c|}
\hline Amostras & Band Gap \\
\hline Filmes $\mathrm{TiO}_{2} 650^{\circ} \mathrm{C}$ & 3,2 \\
\hline Filmes $\mathrm{TiO}_{2} 700 \stackrel{\circ}{\mathrm{C}}$ & 2,91 \\
\hline Filmes $\mathrm{TiO}_{2} 750^{\circ} \mathrm{C}$ & 2,85 \\
\hline Filmes $\mathrm{TiO}_{2} 800 \stackrel{\circ}{\mathrm{C}}$ & 2,82 \\
\hline Filmes $\mathrm{TiO}_{2} / \mathrm{WO}_{3} 650^{\circ} \mathrm{C}$ & 2,58 \\
\hline Filmes $\mathrm{TiO}_{2} / \mathrm{WO}_{3} 700{ }^{\circ} \mathrm{C}$ & 2,57 \\
\hline Filmes $\mathrm{TiO}_{2} / \mathrm{WO}_{3} 750^{\circ} \mathrm{C}$ & 2,56 \\
\hline Filmes $\mathrm{TiO}_{2} / \mathrm{WO}_{3} 800{ }^{\circ} \mathrm{C}$ & 2,54 \\
\hline Filmes $\mathrm{TiO}_{2} / \mathrm{Na}_{2} \mathrm{WO}_{4} \cdot 2 \mathrm{H}_{2} \mathrm{O} 650 \stackrel{\circ}{ }{ }^{\circ} \mathrm{C}$ & 2,52 \\
\hline Filmes $\mathrm{TiO}_{2} / \mathrm{Na}_{2} \mathrm{WO}_{4} \cdot 2 \mathrm{H}_{2} \mathrm{O} \quad 700 \stackrel{\circ}{\circ} \mathrm{C}$ & 2,32 \\
\hline Filmes $\mathrm{TiO}_{2} / \mathrm{Na}_{2} \mathrm{WO}_{4} \cdot 2 \mathrm{H}_{2} \mathrm{O} 750^{\circ} \mathrm{C}$ & 2,27 \\
\hline Filmes $\mathrm{TiO}_{2} / \mathrm{Na}_{2} \mathrm{WO}_{4} \cdot 2 \mathrm{H}_{2} \mathrm{O} 800{ }^{\circ} \mathrm{C}$ & 2,24 \\
\hline Filmes $\mathrm{TiO}_{2}-\mathrm{P}_{25}$ padrão & 3,2 \\
\hline
\end{tabular}

\section{AGRADECIMENTOS}

Os autores agradecem o apoio financeiro da Universidade Federal do Rio Grande do Sul (UFRGS), da Coordenação de Aperfeiçoamento de Pessoal de Nível Superior (CAPES) e do CNPq. 


\section{CONCLUSÕES}

É notório pelos resultados colorimétricos e fotocatalíticos que a presença de tungstênio nas amostras aumenta consideravelmente sua fotoatividade e a capacidade de absorção óptica. Isso porque o tungstênio altera a posição da banda de valência e da banda de condução do $\mathrm{WO}_{3}$ em relação ao $\mathrm{TiO}_{2}$, inibindo a recombinação do par elétron/lacuna permitindo a transferência de cargas entre os dois semicondutores, aumentando a eficiência do processo; também devido à redução do gap de $\mathrm{TiO}_{2}$ e à elevação da temperatura de calcinação, o que ocasionou transições eletrônicas na estrutura das amostras, favorecendo suas propriedades ópticas. Isso pôde ser visto nos filmes sintetizados. O aumento da excitação térmica promoveu elétrons da banda de valência para a banda de condução, causando uma desordem na estrutura eletrônica dos filmes, intensificando a capacidade fotocatalítica e a região de absorção da luz pelos filmes.

\section{REFERÊNCIAS}

[I] Callister, W. D. Jr., Ciência e Engenharia de Materiais: Uma introdução, $5^{\underline{a}}$ edição, LTC, Rio de Janeiro (2002) p.589.

[2] Galvão, J. R.; Scarminio, J. Fotocromismo em filmes finos de óxidos de tungstênio de diferentes composições. Química Nova, 26 (2003) 488-492.

[3] Gonçalves, A. M. F. Desenvolvimento de Tintas Fotocrómicas para Aplicação em Embalagens Inteligentes. 2013, 82p. Dissertação de Mestrado (Faculdade de Ciências e Tecnologia) - Universidade Nova de Lisboa.

[4] Coelho, E. C. de S. Síntese, caracterização e propriedades fotocrômicas de sais derivados do ácido N (3,5 - dinitrobenzoil) - $\alpha$ - fenilglicina. 2008. 78p. Dissertação de Mestrado (Centro de Ciências Exatas e da Natureza- Departamento de Química Fundamental) - Programa de Pós- Graduação em Ciência de Materiais, Universidade Federal de Pernambuco, Recife.

[5] Sobrinho, J. A. Fotocromismo e Luminescência de Compostos a Base de Tungstênio e Íons Terras Raras Trivalentes Via Síntese Hidrotérmica. 2015, 148p. Dissertação de Mestrado (Programa de Pós-Graduação em Química) - Universidade Estadual Paulista, Araraquara. 
[6] Feltrin, J.; Sartor, M. N.; De Noni, A. J; Bernardin, A. M.; Hotza, D.; Labrincha, J. Superfícies fotocatalíticas de titânia em substratos cerâmicos. Parte I: Síntese, estrutura e fotoatividade. Cerâmica, 59 (2013) 620-632.

[7] Lu, X.; Liu, X.; Zhang, W.; Wang, C.; Wei, Y. Large-scale synthesis of tungsten oxide nanofibers by electrospinning. Journal of Colloid and Interface Science, 298 (2006) 996-999.

[8] Feltrin, J. Jr.; Sartor, M. N. A. DE Noni; A. M. Bernardin, D. Hotza, Labrincha, J. A., Superfícies fotocatalíticas de titânia em substratos cerâmicos. Parte I: Síntese, estrutura e fotoatividade, Cerâmica, 2013, v.59, pp.620-632.

[9] Muccillo, E. N. S., Condutores de íons oxigênio - uma breve revisão, Cerâmica, 2008, v.54, Pp.129-I44.

[ro] Bharti, B., Kumar, S., Lee, H. N., Rajesh, K., Formation of oxygen vacancies and $\mathrm{Ti}_{3}+$ state in $\mathrm{TiO}_{2}$ thin film and enhanced optical properties by air plasma treatment, Scientific Reports, 2016, pp.I-I2.

[II] Oliveira, H. G. Eletrodos porosos contendo $\mathrm{TiO}_{2}$ e $\mathrm{WO}_{3}$ : propriedades eletroquímicas e atividade fotocatalítica para remoção do corante rodamina $6 \mathrm{G}$ e do hormônio I7 $\alpha$-etinilestradiol em solução aquosa. 2012. I88p. Doutorado. (Tese de doutorado em Ciências) Instituto de Química da Universidade Estadual de Campinas SP/Campinas.

[12] Chen, S., Xiao, Y., Wang, Y., Hu, Z., Zhao, H., and Xie, W. A Facile Approach to Prepare Black $\mathrm{TiO}_{2}$ with Oxygen Vacancy for Enhancing Photocatalytic Activity. Nanomaterials, 8 (2018) 2-16.

[13] Soares, L. G., Alves, A. K., Analysis of colorimetry using the CIE-L*a*b* system and the photocatalytic activity of photochromic films. Journal Materials Research, 2018, v.105, pp.318-321. 